\title{
A INÉRCIA DA TAXA DE JUROS NA POLÍTICA MONETÁRIA
}

\author{
Fernando de Holanda Barbosa ${ }^{1}$
}

\section{INTRODUÇÃ̃O}

Um fato estilizado no comportamento dos bancos centrais na condução da política monetária consiste no ajuste gradual da taxa de juros, evitando movimentos súbitos e, conseqüentemente, tornando a taxa de juros menos volátil. Esse fenômeno é conhecido na literatura econômica [Goodfriend (1987) e (1991), Mankiw e Miron (1991), Rudebusch (1995), Clarida, Galí e Gertler (1999) e Thornton (2004)], como suavização da taxa de juros (interest rate smoothing) e ele traduz-se na dependência da taxa de juros da sua própria história recente, como descrito, por exemplo, na seguinte equação,

$$
r_{t}=\theta \quad r_{t}^{*}+(1-\theta) r_{t-1}+\varepsilon_{t}, 0<\theta \leq 1
$$

onde a taxa de juros no período $t$ depende da taxa de juros do período $t-1, \theta$ é o peso dado à taxa de juros desejada $r^{*}$, que deve ser especificada de acordo com a regra de política monetária seguida pelo Banco Central, e representa choques estocásticos. Clarida, Galí e Gertler [(1999), p. 1687] reportam que, para dados trimestrais, a ordem de grandeza para o parâmetro $\theta$ fica entre 0,1 e 0,2 , que sugere um ajustamento muito lento e uma inércia muito grande para a taxa de juros.

As razões que determinam esse tipo de comportamento ainda não são bem conhecidas na teoria monetária, e elas não serão analisadas neste trabalho, que parte do pressuposto de que a inércia da taxa de juros é um fato empírico bem documentado. Este artigo tem como objetivo analisar as

Vargas.

1 Professor da Escola de Pós-Graduação em Economia da Fundação Getúlio 
condições que o parâmetro de suavização tem de satisfazer para que a regra de política monetária seja estável.

O trabalho está organizado do seguinte modo: a Seção 2 analisa a estabilidade de um modelo que contém três ingredientes, uma regra de política monetária à la Taylor, uma curva IS e uma curva de Phillips com nível de preços predeterminado e inércia na taxa de inflação; a Seção 3 analisa a dinâmica do modelo da seção anterior quando adiciona-se a hipótese de inércia na taxa de juros por meio de um mecanismo de ajustamento parcial; a Seção 4 analisa a questão de inércia da taxa de juros num modelo novo-keynesiano com a curva de Phillips, que supõe o nível de preços predeterminado, mas no qual a taxa de inflação pode mudar instantaneamente de valor; a Seção 5 conclui o trabalho.

\section{REGRA DE POLÍTICA MONETÁRIA E DINÂMICA DE ESTABILIZAÇÃO}

A curva IS supõe que o hiato do produto depende da diferença entre as taxas de juros real de curto $\rho$ e de longo prazo $\bar{\rho}$, de acordo com:

$$
y-\bar{y}=-\alpha(\rho-\bar{\rho}), \alpha>0
$$

onde y é o logaritmo do produto real e $\bar{y}$ é o logaritmo do produto potencial.

O Banco Central fixa a taxa de juros nominal por meio da regra de política monetária de Taylor, que depende da taxa de juros real de longo prazo, da taxa de inflação $\pi$, da diferença entre a taxa de inflação e a meta da taxa de inflação $\bar{\pi}$, e do hiato do produto:

$$
r=\bar{\rho}+\pi+\beta(\pi-\bar{\pi})+\gamma(y-\bar{y}), \beta>0, \gamma>0
$$

A curva de Phillips é uma curva aceleracionista, na qual a aceleração da inflação é proporcional ao hiato do produto:

$$
\dot{\pi}=\delta(y-\bar{y}), \delta>0
$$

Substituindo-se o hiato do produto da curva IS na curva de Phillips, obtém-se:

$$
\dot{\pi}=-\alpha \delta(\rho-\bar{\rho})
$$


A combinação da curva IS com a regra de política monetária produz a seguinte equação da taxa de inflação em função da taxa de juros real:

$$
\pi=\bar{\pi}+\frac{\beta}{1+\alpha \gamma}(\rho-\bar{\rho})
$$

FIGURA 1

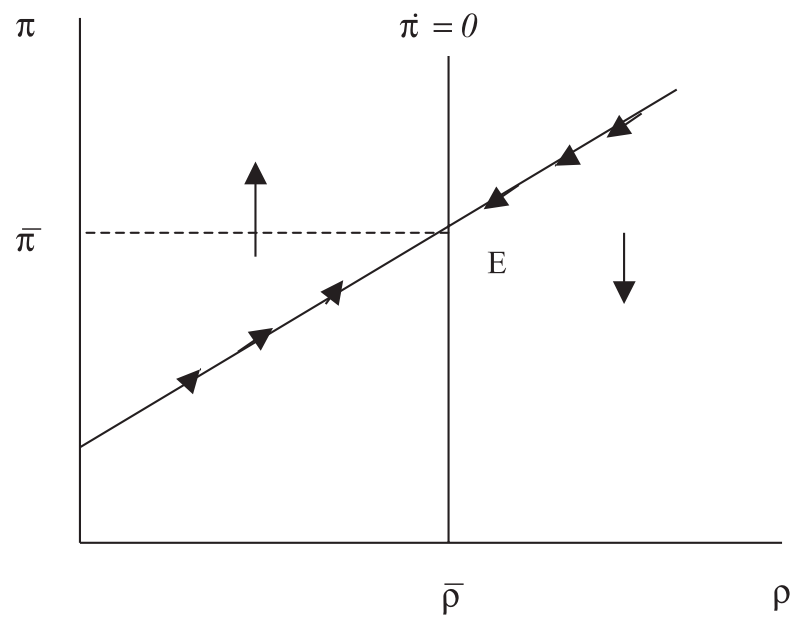

O diagrama de fases da Figura 1, com a inflação medida no eixo vertical e a taxa de juros real no eixo horizontal, mostra a dinâmica do modelo formado pelas equações (5) e (6). Quando a taxa de juros real for maior do que a taxa de juros real de longo prazo, a inflação diminui. Caso contrário, ela aumenta. A regra de política monetária é estável, pois conduz a economia ao pleno emprego e a meta de inflação desejada pelo Banco Central.

Considere agora o seguinte experimento de política econômica: o Banco Central decide no instante zero reduzir a meta da taxa de inflação de $\bar{\pi}_{0}$ para $\bar{\pi}_{1}$, como descrito na Figura 2. Essa é uma mudança permanente e não antecipada pelo público. 


\section{FIGURA 2}

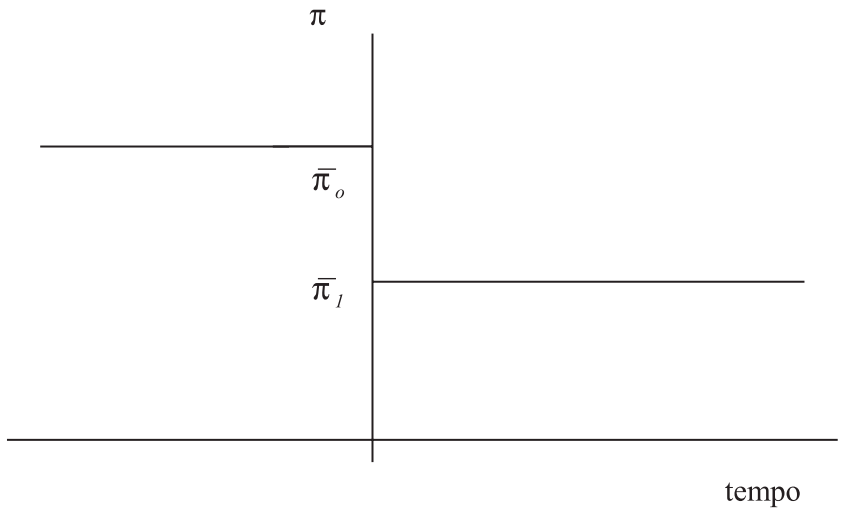

FIGURA 3

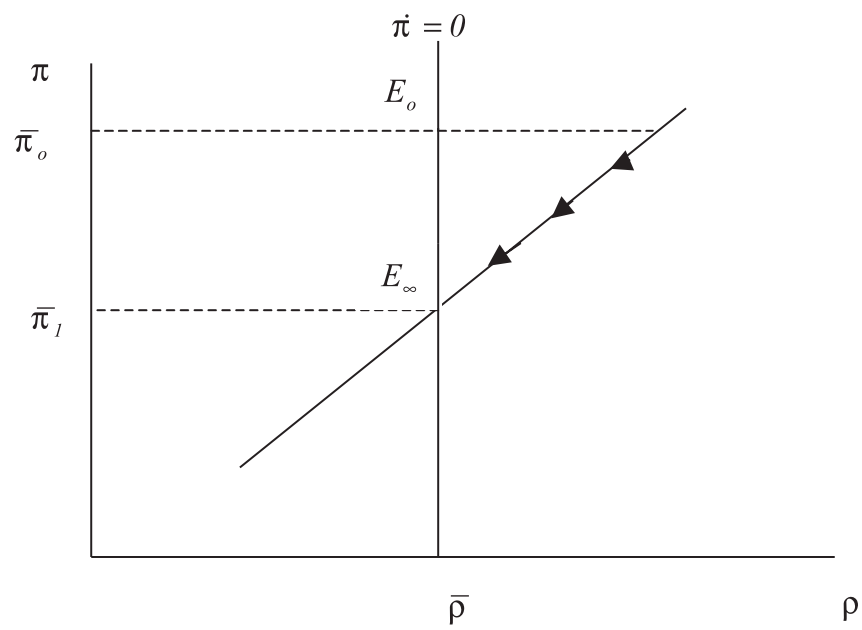


A Figura 3 mostra a dinâmica de ajustamento da economia quando a nova política é introduzida. Como a taxa de inflação no momento inicial é predeterminada, a taxa de juros real aumenta instantaneamente de valor, produzindo um processo recessivo que acarreta a redução da taxa de inflação. A taxa de juros real e a taxa de inflação convergem gradualmente para o novo equilíbrio de longo prazo, quando a nova meta de inflação é alcançada.

\section{A INÉRCIA DA TAXA DE JUROS}

A inércia da taxa de juros é descrita por um mecanismo de ajustamento parcial, em que a variação da taxa de juros é proporcional à diferença entre a taxa de juros desejada e a taxa de juros atual. ${ }^{2}$ Isto é:

$$
\dot{r}=\theta\left(r^{*}-r\right), \theta>0
$$

Essa equação é equivalente à equação (1) para o modelo escrito em variáveis contínuas. Ela supõe que existe algum custo de ajustamento que impede o banco central de fixar imediatamente a taxa de juros nominal no nível desejado. Quando o parâmetro $\theta \rightarrow \infty$ o ajustamento é instantâneo. Caso contrário, a taxa de juros ajusta-se gradualmente para sua posição de equilíbrio. A taxa de juros desejada segue a regra de Taylor da seção anterior, repetida aqui por conveniência,

$$
r^{*}=\bar{\rho}+\pi+\beta(\pi-\bar{\pi})+\gamma(y-\bar{y})
$$

Com um pouco de álgebra, as duas equações anteriores resultam na equação diferencial para a taxa de juros real:

$$
\dot{\rho}=\beta \theta(\pi-\bar{\pi})-[(1+\alpha \gamma) \theta-\alpha \delta](\rho-\bar{\rho})
$$

O modelo formado pelas equações (5) e (9) tem a seguinte matriz jacobiana:

2 O Apêndice mostra como esse mecanismo de ajustamento parcial pode ser derivado quando o agente econômico minimiza o valor presente do custo de ajustamento para a mudança de uma variável. 


$$
J=\left[\begin{array}{ll}
\frac{\partial \dot{\pi}}{\partial \pi} & \frac{\partial \dot{\pi}}{\partial \rho} \\
\frac{\partial \dot{\rho}}{\partial \pi} & \frac{\partial \dot{\rho}}{\partial \rho}
\end{array}\right]=\left[\begin{array}{cc}
0 & -\alpha \delta \\
\beta \theta & -[(1+\alpha \gamma) \theta-\alpha \delta]
\end{array}\right]
$$

O determinante dessa matriz é positivo:

$$
|J|=\alpha \beta \delta \theta>0
$$

O traço da matriz $J$ tanto pode ser positivo como negativo. Para que o sistema dinâmico formado pelas equações (5) e (9) seja estável, o traço tem de ser negativo. Isso significa dizer que o parâmetro $\theta$ de ajuste da taxa de juros tem de satisfazer a restrição:

$$
\operatorname{tr} J<0 \text { se } \theta>\underline{\theta}=\frac{\alpha \delta}{1+\alpha \gamma}
$$

A conclusão a que se chega com essa restrição é que o Banco Central não pode ser muito lento em ajustar a taxa de juros quando houver uma mudança na taxa de juros desejada. A estabilidade do modelo impõe um piso ao parâmetro $\theta$ de ajuste da taxa de juros, que depende do parâmetro $\alpha$ da curva IS, do parâmetro $\delta$ da curva de Phillips e do parâmetro $\gamma$ da regra de política monetária. Quanto maior os dois primeiros, menor a inércia da taxa de juros. Quanto maior a resposta da política monetária ao hiato do produto, menor o piso do coeficiente de ajuste da taxa de juros.

A existência de um piso para o coeficiente $\theta$ significa dizer que na especificação da equação (1) existe um limite superior para o grau de suavização, ou de inércia, da taxa de juros pelo Banco Central. Os parâmetros da economia e da regra de política monetária impõem uma restrição ao comportamento do Banco Central no processo de suavização da taxa de juros. O diagrama de fases da Figura 4 mostra a dinâmica do modelo, existindo quatro regiões com diferentes movimentos das taxas de inflação e de juros real, caso a economia não esteja no ponto $E$ de equilíbrio de longo prazo. 
FIGURA 4

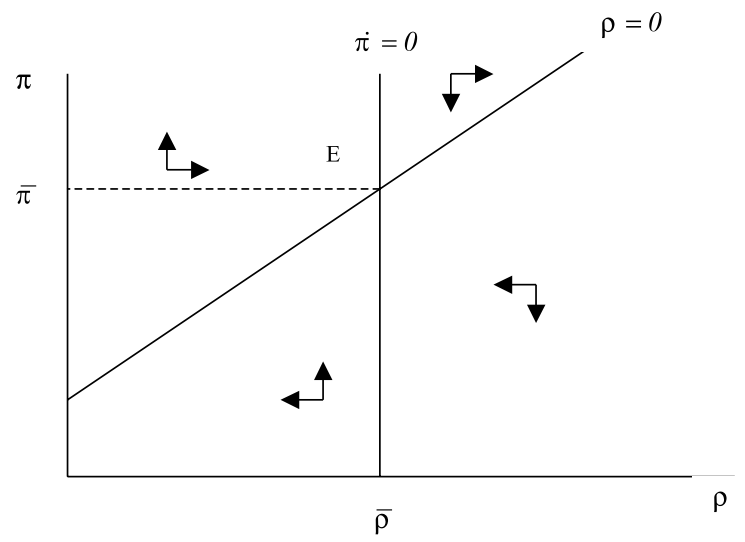

A Figura 5 descreve a dinâmica de ajustamento da economia quando o Banco Central decide mudar a meta de inflação, no experimento de política monetária descrito na Figura 2. A taxa de inflação começa a cair gradualmente, enquanto a taxa de juros real aumenta até atingir seu nível máximo no ponto em que a trajetória da economia corta a reta em que $\dot{\rho}=0$. A taxa de inflação continua a declinar, atingindo depois de um certo período um valor menor do que aquele que corresponde ao de equilíbrio de longo prazo (no conhecido fenômeno de undershooting), voltando a subir até convergir para a nova meta de inflação. A economia tem uma trajetória recessiva desde a mudança da política monetária até a taxa de inflação atingir o seu menor valor, quando começa a ocorrer um período de aquecimento, com o produto real ultrapassando o produto potencial, em virtude da taxa de juros real ficar abaixo, durante um certo intervalo de tempo, do seu valor de equilíbrio de longo prazo. 
FIGURA 5

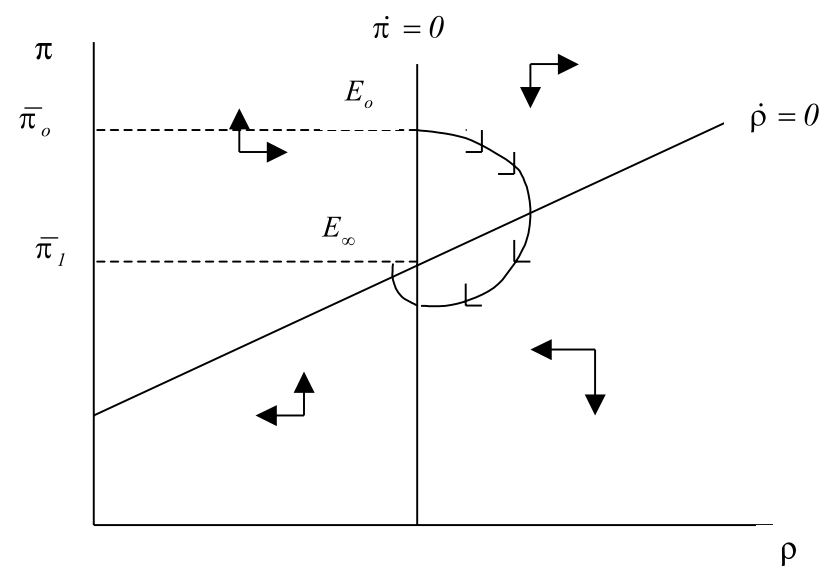

\section{INÉRCIA DA TAXA DE JUROS NO MODELO NOVO- KEYNESIANO}

No modelo novo-keynesiano, o nível de preços é predeterminado, mas a taxa de inflação pode mudar repentinamente. A curva de Phillips à la Calvo é proporcional ao hiato do produto, mas o coeficiente de proporcionalidade é negativo,

$$
\dot{\pi}=-\phi(y-\bar{y}), \phi>0
$$

A curva IS, a regra de política monetária e o mecanismo de ajustamento parcial da taxa de juros são os mesmos da seção anterior. Neste caso, o modelo fica mais fácil de ser analisado por meio de um sistema dinâmico com as variáveis taxa de inflação e taxa de juros nominal. Com um pouco de álgebra, chega-se ao seguinte sistema de equações diferenciais:

$$
\begin{gathered}
\dot{\pi}=-\alpha \phi(\pi-\bar{\pi})+\alpha \phi(r-\bar{r}) \\
\dot{r}=-(1+\alpha \gamma) \theta(r-\bar{r})+\theta(\beta+1+\alpha \gamma)(\pi-\bar{\pi})
\end{gathered}
$$


onde $\bar{r}=\bar{\rho}+\bar{\pi}$ é a taxa de juros nominal de longo prazo. A matriz jacobiana desse sistema é dada por:

$$
J=\left[\begin{array}{ll}
\frac{\partial \dot{\pi}}{\partial \pi} & \frac{\partial \dot{\pi}}{\partial r} \\
\frac{\partial \dot{r}}{\partial \pi} & \frac{\partial \dot{r}}{\partial r}
\end{array}\right]=\left[\begin{array}{cc}
-\alpha \phi & \alpha \phi \\
\theta(\beta+1+\alpha \gamma) & -(1+\alpha \gamma) \theta
\end{array}\right]
$$

O determinante dessa matriz é negativo,

$$
|J|=-\alpha \beta \theta \phi<0
$$

e o ponto de equilíbrio desse sistema é um ponto de sela. Logo, não existe qualquer restrição sobre o parâmetro $\theta$ de ajustamento da taxa de juros. A Figura 6, com a taxa de juros nominal no eixo vertical e a taxa de inflação no eixo horizontal, mostra o diagrama de fases desse modelo. Na sela desse modelo, a taxa de juros nominal e a taxa de inflação são negativamente correlacionadas.

FIGURA 6

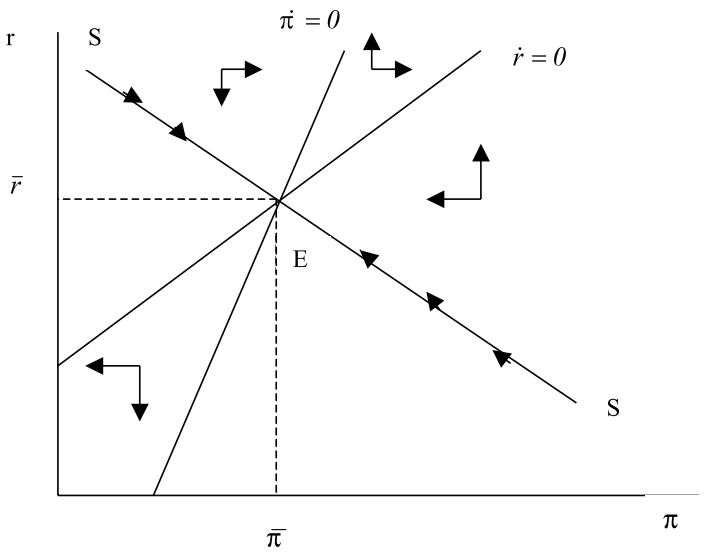


A Figura 7 descreve o que acontece com a taxa de juros nominal e com a taxa de inflação num experimento de política monetária em que o Banco Central reduz permanentemente, sem anúncio prévio, a meta da taxa de inflação, como descrito na Figura 2. A taxa de inflação sofre uma redução instantânea, porém a taxa de juros nominal é uma variável predeterminada, que ajusta-se gradualmente para o seu novo valor de equilíbrio de longo prazo.

FIGURA 7

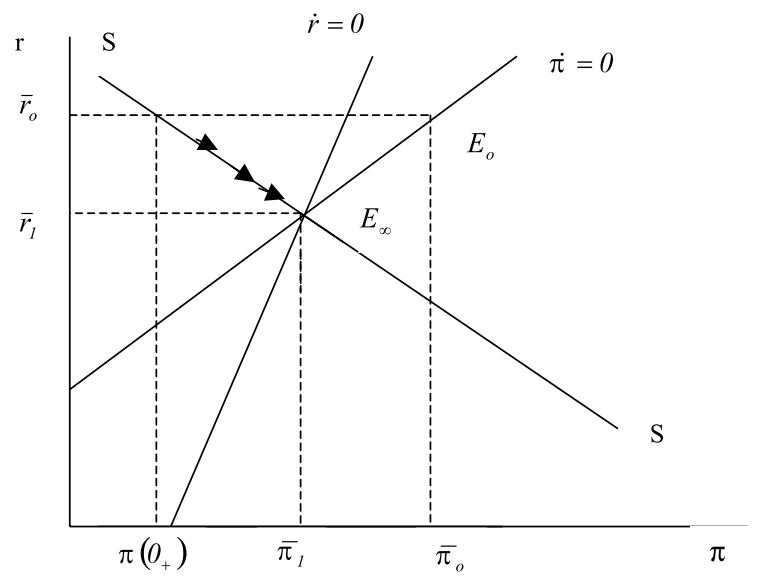

\section{CONCLUSÃO}

Este trabalho mostrou que num modelo em que existe inércia na taxa de inflação, o Banco Central não pode se dar ao luxo de ajustar a taxa de juros nominal da economia na velocidade que desejar, pois há um limite superior para o coeficiente de inércia. Caso contrário, a regra de política monetária de Taylor produz um regime monetário instável.

No modelo novo-keynesiano não existe qualquer restrição sobre o grau de inércia da taxa de juros. O Banco Central neste caso pode escolher de maneira discricionária a velocidade de ajuste da taxa de juros. Todavia, a dinâmica da economia quando submetida a experimentos de política monetária produz resultados que não são consistentes com a evidência empírica. Uma 
redução da meta de inflação não antecipada acarreta uma redução instantânea da taxa de inflação, com um undershooting da taxa de inflação, e depois uma subida gradual da taxa de inflação para o seu novo equilíbrio de longo prazo.

\section{APÊNDICE}

Admita-se que o custo de ajustamento de uma variável $y$ é uma função quadrática da diferença entre o seu valor corrente e o valor da mesma em equilíbrio, e de sua taxa de variação, de acordo com,

$$
\int_{0}^{\infty} e^{-\rho t}\left[\frac{1}{2}\left(y-y^{*}\right)^{2}+\frac{\alpha}{2} \dot{y}^{2}\right] d t
$$

onde $\rho$ é a taxa de juros real usada para descontar o fluxo do custo de ajustamento. O problema consiste em escolher a trajetória da variável y de sorte a minimizar a expressão acima. Esse problema pode ser resolvido pela teoria do controle ótimo, introduzindo-se a variável $x$ como variável de controle. Isto é, minimizar,

$$
\int_{0}^{\infty} e^{-\rho t}\left[\frac{1}{2}\left(y-y^{*}\right)^{2}+\frac{\alpha}{2} x^{2}\right] d t
$$

com a condição de que a restrição seja satisfeita,

e supondo,

$$
\dot{y}=x
$$

$$
y(0) \text { dado }
$$

O Hamiltoniano desse problema é dado por:

$$
H=\frac{1}{2}\left(y-y^{*}\right)^{2}+\frac{\alpha}{2} x^{2}+\lambda x
$$

As condições de primeira ordem para um mínimo são:

$$
\frac{\partial H}{\partial x}=\alpha \quad x+\lambda=0
$$


e

$$
\dot{\lambda}=\rho \lambda-\frac{\partial H}{\partial y} \quad, \quad \frac{\partial H}{\partial y}=y-y^{*}
$$

A trajetória ótima tem de satisfazer a condição de transversalidade:

$$
l i m_{t \rightarrow \infty} \lambda y e^{-\rho t}=0
$$

As duas condições de primeira ordem são equivalentes ao seguinte sistema de equações diferenciais:

$$
\begin{gathered}
\dot{y}=-\frac{1}{\alpha} \lambda \\
\dot{\lambda}=\rho \lambda-\left(y-y^{*}\right)
\end{gathered}
$$

A matriz jacobiana desse sistema é dada por:

$$
J=\left[\begin{array}{ll}
\frac{\partial \dot{y}}{\partial y} & \frac{\partial \dot{y}}{\partial \lambda} \\
\frac{\partial \dot{\lambda}}{\partial y} & \frac{\partial \dot{\lambda}}{\partial \lambda}
\end{array}\right]=\left[\begin{array}{cc}
0 & -\frac{1}{\alpha} \\
-1 & \rho
\end{array}\right]
$$

O determinante dessa matriz é negativo,

$$
|J|=-\frac{1}{\alpha}<0
$$

e o ponto de equilíbrio do sistema é um ponto de sela. A Figura A1 mostra o diagrama de fases do sistema com a variável $y$ no eixo horizontal e a variável de coestado $\lambda$ no eixo vertical. Os pontos que correspondem a $\dot{y}=0$ coincidem com o eixo horizontal, e a trajetória de sela SS é positivamente inclinada. 


\section{FIGURAA1}

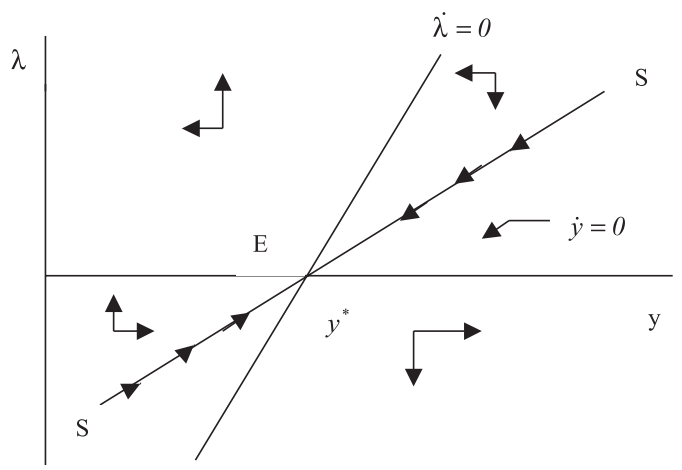

A Figura A2 mostra a trajetória de ajustamento quando a variável y muda seu valor de equilíbrio, de $y_{0}^{*}$ para $y_{1}^{*}$. A variável $y$ não muda repentinamente de valor, mas a variável $\lambda$ ajusta-se instantaneamente, produzindo uma trajetória em que a variável y converge gradualmente para seu novo valor de equilíbrio no caminho de sela indicado na figura.

\section{FIGURA A2}

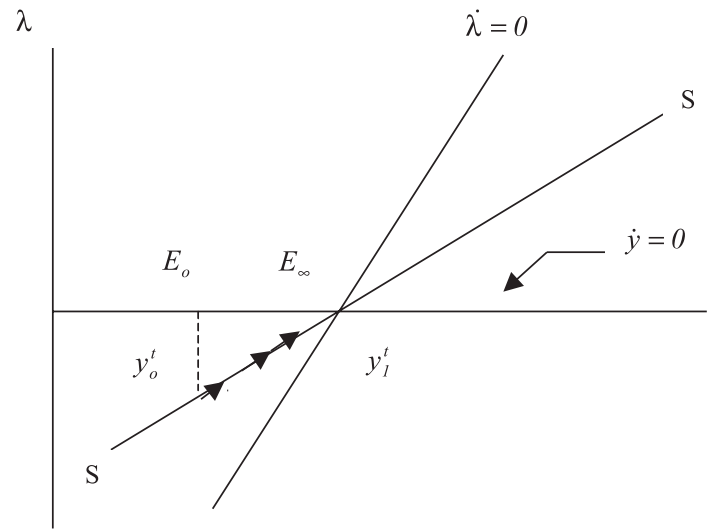

A equação de $y$ que corresponde à sela é dada por:

$$
y=y^{*}+C e^{-\mu t}, \mu>0
$$


onde $C$ é uma constante, e $\mu$ é uma raiz da equação homogênea,

$$
\ddot{y}-\rho \quad \dot{y}-\frac{1}{\alpha} y=0
$$

cujo valor é:

$$
\mu=\frac{\rho+\sqrt{\rho^{2}+\frac{4}{\alpha}}}{2}
$$

Derivando-se a equação de y com relação ao tempo, é fácil verificarse que o mecanismo de ajustamento parcial depende do parâmetro do custo de mudança da variável y. Isto é:

$$
\dot{y}=\frac{1}{\sqrt{\alpha}}\left(y-y^{*}\right)
$$

onde admitiu-se, por simplicidade, que a taxa de juros real é igual a zero. Logo, se o parâmetro $\alpha$ for igual a zero, o ajustamento é instantâneo. Caso contrário, o ajustamento é parcial.

\section{RESUMO}

Um fato estilizado no comportamento dos bancos centrais consiste na suavização da taxa de juros, na qual o reajuste da taxa de juros depende de sua própria história recente. Este trabalho tem como objetivo analisar as condições que o parâmetro de suavização tem de satisfazer a regra de política monetária estável. A análise é feita em dois modelos, num existe inércia da taxa de inflação e no outro a taxa de inflação pode mudar instantaneamente de valor. Palavras-chave: política monetária, regra de taxa de juros, inércia da taxa de juros. 


\begin{abstract}
A stylized fact of central bank behavior is interest rate smoothing. This paper takes this fact as given and analyses the conditions that the smoothing parameter of the monetary policy rule has to attend for the model to be stable. This analysis is done in two models, one with inflation inertia and the other one with inflation rate as a jump variable.

Key-words: monetary policy, monetary policy rule, interest rate smoothing.
\end{abstract}

\title{
REFERÊNCIAS
}

CLARIDA, Richard. GALÍ, J.; GERTLER, M. The Science of Monetary Policy: A Keynesian Perspective. Journal of Economic Literature, n. 37, p. 1661-1707, 1999. GOODFRIEND, M. Interest Rate Smoothing and Price Level Trend-Stationary. Journal of Monetary Economics, n. 19, p. 335-348, 1987.

GOODFRIEND, M. Interest Rates and the Conduct of Monetary Policy. CarnegieRochester Conference Series on Public Policy, n. 34, p. 7-30, 1991.

MANKIW, N. Gregory; MIRON, Jeffrey A. Should the FED Smooth Interest Rates? The Case of Seasonal Monetary Policy. Carnegie-Rochester Conference Series on Public Policy, n. 34, p. 41-70, 1991.

RUDEBUSCH, G. D. Federal Reserve Interest Rate Targeting, Rational Expectations and the Term Structure. Journal of Monetary Economics, n. 35, p. 245-274, 1995.

THORNTON, D. L. The Fed and Short-Term Rates: Is it Open Market Operations, Open Mouth Operations or Interest Rate Smoothing? Journal of Banking \& Finance, n. 28, p. 475-498, 2004. 